\title{
Preface: Special Session SpS7 The impact hazard: current activities and future plans
}

In 2012, at the time of the Beijing GA, two decades had passed since the publication of the Spaceguard Report. Time has not passed in vain for the subject of NEO-related hazards, and we are currently in a totally different situation than in the early nineties. The amount of work done, and the level of awareness of the underlying problems, have both risen to such a level that a re-assessment by the astronomical community of its rôle and involvement was in order, and the GA Special Session 7 "The impact hazard: current activities and future plans" was aimed exactly at that.

Increasingly sophisticated sky surveys have succeeded in discovering thousands of NEOs, and much has been learned about the physical properties of two NEOs, Eros and Itokawa, from the NEAR-Shoemaker and Hayabusa rendezvous missions. However, while observational and modeling work aimed at investigating the physical nature of the NEO population as a whole is gathering pace, efforts in this area are still being outpaced by the discovery rate.

Groundbased NEO observational efforts suffer from the preference of telescope timeallocation committees for purely scientifically motivated proposals. The space-based observational programs Warm Spitzer and WISE are providing size, albedo, and potentially other mitigation relevant data for hundreds of NEOs but both are limited, short-lived programs. Progress in other (more expensive) areas, such as developing and testing mitigation techniques, has been more limited.

However, events like the Chelyabinsk superbolide of 15 February 2013, with its vast media coverage and worldwide echo, do remind us that the NEO hazard is a field that continues to require our attention.

G. B. Valsecchi, A. Milani and W. Huebner, co-chairs SOC 